Penultimate Draft - Please cite only the final published version

Synthese (2020). Doi: 10.1007/s11229-020-02594-3

\title{
Integrated-Structure Emergence and Its Mechanistic Explanation
}

\author{
Gil Santos ${ }^{1}$
}

\begin{abstract}
This paper proposes an integrated-structure notion of interlevel (part-whole) emergence, from a dynamic relational ontological perspective. First, I will argue that only the individualist essentialism of atomistic metaphysics can block the possibility of interlevel emergence. Then I will show that we can make sense of emergence by recognizing the formation of structures of transformative and interdependent causal relations in the generation and development of a particular class of mereological complexes called integrated systems. Finally, I shall argue that even though the emergent structural attributes of such systems are not micro-determined nor microreducible, they can still be accounted for by an interlevel integrative neo-mechanistic form of explanation.
\end{abstract}

Keywords: emergence; integrated systems; dynamic relational ontology; transformative relations; internal relations; structure; mechanistic explanation.

\footnotetext{
1 'Departamento de História e Filosofia das Ciências' and 'Centro de Filosofia das Ciências da Universidade de Lisboa' at 'Faculdade de Ciências da Universidade de Lisboa', Campo Grande, C4.3.24, 1749-016 Lisboa. I acknowledge the financial support of FCT, 'Fundação para a Ciência e a Tecnologia, I.P.' (Stimulus of Scientific Employment, Individual Support 2017: CEECIND/03316/2017). This work is part of the FCT Project 'Emergence in the Natural Sciences: Towards a New Paradigm' (PTDC/FERHFC/30665/2017).
} 


\section{Introduction}

It is a well-known fact that the notion of emergence has no univocal meaning among philosophers, and that both philosophers and scientists have been using different notions of emergence, either to support or to refute it (Hendry, Gibb \& Lancaster 2019: 2-3). Still, I assume we can all agree that any naturalistic notion of emergence may be minimally characterized in terms of a relation of both partial dependence and partial independence (the latter being conceived in terms of a relative irreducibility or autonomy) between a given entity (the putative 'emergent') and the entities with respect to which it may be said to be emergent (the 'emergence base') ${ }^{2}$.

Nonetheless, this is only a minimal characterization. Therefore, it is crucial to spell out in the clearest possible way what notion of emergence one intends to deal with, since refuting or endorsing a particular notion does not necessarily imply the denial or approval of any other notion.

In this paper I will address ontological emergence as an interlevel relation between parts and wholes, in terms of their respective qualitative properties, causal powers or actual activities. (Throughout this paper, I shall use 'attribute' as an umbrella term for those types of features). Specifically, emergence will be considered as a relation between certain attributes instantiated by a composed system taken as a structured whole, and some compositional relation between the attributes instantiated by that system's compositionally or historically associated lower-level entities - that is, by the entities that are its contemporary proper parts in a given period of time, or by the precursor entities of such parts.

This paper is structured as follows. Section 2 presents the basic problems involved in the notion of ontological emergence as an interlevel or part-whole relation. In section 3 I will argue that only the individualist essentialism of atomistic metaphysics can block the possibility of interlevel emergence, because only on the basis of such a view are micro-determinism and micro-reductionism proven to be universally valid

\footnotetext{
${ }^{2}$ See, for example, Mark Bedau and Paul Humphrey's characterizations of emergence: “(1) Emergent phenomena are somehow constituted by, and generated from, underlying processes; (2) Emergent phenomena are somehow autonomous from underlying processes" (Bedau 1997: 376); "Emergence is, broadly speaking, the view that there are features of the world - objects, properties, laws, perhaps other things - that are manifested as a result of the existence of other, usually more basic, entities but that cannot be completely reduced to those other entities" (Humphreys 2006: 90).
} 
doctrines. In section 4 , building on and expanding an approach already elaborated in (Santos 2015a), I will put forward a notion of interlevel emergence based on a dynamic relational ontological view and, in particular, on the existence of transformative and interdependent causal relations. In section 5, I will argue that such kind of interlevel emergence can be found in the generation and development of a particular class of mereological complexes called integrated systems, and I will briefly consider, as a prototypical example, the formation of the structural and functional identity of eukaryotic cells. Finally, I will show why emergent structural and functional attributes of integrated systems are neither micro-determined nor micro-reducible, although they can be accounted for by an interlevel integrative neo-mechanistic form of explanation.

\section{The Notion of Interlevel Emergence}

The reason why the notion of emergence has acquired an undeniable importance in the philosophy of science is that it has always been defined, in opposition to some notion of ontological and/or epistemological reduction, as a relation between certain wholes and their parts, where these entities belong to different levels of organization and different stages of development or evolution. In this respect, it is easy to understand why the debate around the concept of emergence has been so frequently connected to the analysis of different kinds of relations and modes of composition among parts and wholes. Lloyd Morgan famously wrote that emergence involves a "new kind of relation or set of relations" at each ascending level of organization and step of evolution (Morgan 1923: 19, 64). Samuel Alexander identified emergence with the occurrence of new qualities that are distinctive of high-level complexes (Alexander 1979: 45). And Broad addressed the explanation of part-whole relations in terms of general and specific laws of composition and interaction, taking into account both how the parts would behave separately and the laws according to which the behavior of the separate parts is compounded when they act together (Broad 1925: 45-67).

As it is well known, British Emergentists tried to build an alternative to Vitalism, while also pointing to the limits of classical mechanistic reductionism - the 'Ideal Pure Mechanism', as Broad (1925: 44-52) called it -, which led Morgan to explicitly criticize the model of composition and explanation based on the principle of 'algebraic summation' (Morgan 1923: 8). This principle corresponds to what Stuart Mill had previously called the linear principle of composition of causes by way of analogy with 
the classical linear superposition principle of the composition of forces, in which "the joint effect of several causes is identical with the sum of their separate effects" (Mill 1974: 371). Hence, the fundamental problem: should we assume that all levels of organization of reality are reducible, level by level, to the most fundamental set of properties, relations and laws of the putative ultimate physical domain? Or must we recognize that certain levels of organization, although necessarily dependent on underlying or historically previous levels, can acquire a relative degree of autonomy? As Kim once put the issue:

"How are the characteristic properties of a given level related to the properties at the adjacent levels - in particular, to those at the lower levels? How are biological ('vital') properties related to physicochemical properties? How are consciousness and intentionality related to biological/physical properties? How are social phenomena, phenomena characteristic of social groups, related to phenomena involving individual members?" (Kim 1998: 16).

The fundamental role that the assessment of the different kinds of part-whole relations and modes of compositions played in the opposition between classical emergentism and mechanistic reductionism is well exemplified by the fact that the first criticisms of emergence theories, raised by philosophers such as Ernest Nagel and Carl Hempel, were settled in the context of a debate informed by the advent of Gestalt theory (e.g., Grelling \& Oppenheim 1988 [1939]; Rescher \& Oppenheim 1955), General system theory (as developed by Bertalanffy) and Organismic Biology (e.g., Hempel 1951, 1965; Nagel 1951, 1952, 1961).

Nagel understood emergence as a thesis about the "hierarchical organization of things and processes, and the consequent occurrence of properties at 'higher' levels of organization", as well as about evolution taken as a process through which "more complex and 'irreducibly novel' traits and structures" are formed as "unpredictable novelties" (Nagel 1961: 366-367). For this reason, Nagel was led to analyse the different meanings that may be assigned to the notions of 'whole' and 'sum', and the epistemological significance that can be ascribed to the distinction - in the light of the 'additive point of view' - between mechanical 'aggregates' and 'organic unities' (1961: 380-397). Likewise, Hempel noted that the concept of emergence had been traditionally characterized in terms of certain systems' properties that are characterized as 'novel' 
vis-à-vis the information about their parts or constituents. Still, as Hempel observed, in order to evaluate whether a system's property is emergent or not relative to its parts whether in terms of explanation, predictability or inference - several issues have to be firstly clarified, such as what is the class of properties relatively to which a systemic property can be said to be emergent (Hempel 1965: 260-261). This is precisely the core set of problems that I will address.

\section{Micro-reductionism and Atomistic Metaphysics}

If emergence has always been defined against the backdrop of some notion of reduction, we must first clarify which specific notion of reduction it opposes. Indeed, which notion of irreducibility can a notion of emergence tolerate without falling back either to the assertion that there are natural processes that must be accepted as 'brute' ontic facts, fundamentally inaccessible to human explanation, or to the trivial claim of historically variable epistemic limits to our knowledge? In short, under which conditions can an ontologically relevant notion of emergence constitute a positive and consequential epistemological stance?

From the point of view of interlevel or part-whole relations, there are only two possible forms of an absolutely unilateral reduction, insofar as we conceive reduction as a real asymmetric relation between a reducing and a reduced term, namely, microreduction and macro-reduction (Mahner \& Bunge, 1997: 110-117). That is, either we endorse a macro-reduction of the individual attributes of the parts to certain attributes only instantiated by their whole, or we endorse a micro-reduction of the whole's attributes to some composition of the intrinsic attributes of its parts. There is no other option. We must keep in mind that, in order to constitute an absolutely unilateral reduction, the reducing term must have all the resources required to account for the reduced term independently of it. The first possibility is advocated by extreme holism. In such a view, parts are ultimately conceived to be the result of a dismantling of their wholes, while the wholes are seen as primitive or self-imposed entities that come about in a manner one knows not how to account for. In short, wholes structure, but are not structured by, their parts. Hence, parts' attributes are seen as determined or comprised by their wholes' attributes. The opposite view is atomism. If conceived in a fully coherent way, micro-reductionism is a doctrine according to which any system's macro-attribute is reducible (if not in practice, at least in principle) to a certain 
composition relation between some parts' intrinsic attributes, i.e., attributes which the lower-level entities instantiate independently of being interrelated parts of such wholes (e.g., Hüttemann 2004: 34-36; Garson 2006). If conceived as a universally applicable doctrine, micro-reductionism can only thus be grounded in the micro-determinism that follows from the individualistic essentialism of atomistic metaphysics.

The fundamental principle of atomistic metaphysics says that the qualitative and causal identity of every entity is completely determined by its own intrinsic attribute types - exogenous relations can only cause quantitative changes in its already-always possessed intrinsic attribute types ${ }^{3}$. In other words, the qualitative and causal identity of any entity must be completely independent from its exogenous relations with other entities, including in the context of higher-level system's relational structures. The individualistic essentialism of atomistic metaphysics thus expresses an intrinsicnessprinciple of identity.

From this principle follows the micro-reductionist doctrine: any system, as a mereological complex, is completely determined by, and thus reducible to some composition, arrangement or configuration of the intrinsic attribute types instantiated by its historically or constitutively associated lower-level entities. This is the essence of what Humphreys has called 'generative atomism' (2016: 1-3, 11-15), and which is well exemplified in the metaphysics of philosophers such as Lewis (1986: ix-x; 1994: 473), Kim (1993; 1998) or Armstrong (1997).

The "Democritean atomistic doctrine" can thus be said to ground the view that "the world is the way it is because the microworld is the way it is" (Kim 1998: 18). Even if higher-level regularities, given their excessive complexity, "are not reducible to physics in polynomial time", the behavior of any higher-level system "is nevertheless the sum of the behavior of the ultimate physical constituents" (Quine 2008 [1986]: 166). Microdeterminism thus constitutes "the metaphysical basis of the method of microreduction and micro-explanation" (Kim 1993: 102). Any whole or higher-level organization is supposed to be micro-determined, and therefore micro-explained, by the

\footnotetext{
${ }^{3}$ Following Auyang, I take here the notion of 'quantitative' as having a more general meaning than that regarding amount or size: "An individual has various characters, the summary of which is called the individual's state. The general concept of a character can be analyzed into two elements, a type and a value. Blue and red are values of the character type color; big and bigger, values of size. Other characters are temperature, weight, and annual income; their values $-25{ }^{\circ} \mathrm{C}, 250 \mathrm{Kg}$, and $\$ 25,000$ - are the definite predicates attributed to individuals, and their types are represented in the units: degrees Celsius, kilogram, and dollar" (Auyang 1999: 49).
} 
lower level organization of its proper parts (Klee 1984: 44). Micro-determinism must thus exclude any mutual determination between parts and wholes.

In a similar way, Oppenheim and Putnam associated micro-reductionism to the "Democritean" methodology, and to the "possibility that all science may one day be reduced to micro-physics" (Oppenheim \& Putnam, 1958: 16, 27). In this sense, it may be stated that the microphysical order has "total hegemony" over the non-microphysical (Pettit 1993: 220-221), or that the ambition of micro-physicalists is to provide a "microexplanation" of the "behavior of all objects in terms of micro-level properties and relations and micro-level laws" (Clarke 1999: 309) 4

From this it follows that no system's property can ever be emergent, since it can always be represented as a mere combinatorial product of the intrinsic micro-properties, and respective micro-relations, of that system's parts. This corresponds to the standard atomistic-combinatorial notion of 'structural property', first proposed by Armstrong (1978: 69; 1997: 153) and later adopted, among many others, by Kim (1998, 1999). According to that notion, any higher-level system's property is a micro-based property, in the sense of being completely decomposable into, or micro-reducible in terms of, a configuration (as an 'external relation') of the intrinsically determined microconstituents (Armstrong 1978: 69; Kim 1999: 7; Kim 1998: 84). No system's part will ever acquire new relevant properties or causal powers by virtue of its relations within that system, since the structure of any system "can be exhaustively described" in terms of the "intrinsic properties", and respective relations, of its lower-level parts (Kim 1999: 6). Unsurprisingly, the intrinsicness-principle of identity is also at the heart of Kim's (1999) functionalist version of micro-reduction. Indeed, the lower-level realizers of a functionalized macro-property can be addressed without considering the possibility that certain lower-level entities may only instantiate such realizer properties by virtue of interacting in particular higher-level relational structures. That is why you just need to localize the lower-level realizers, and then find some lower-level theory able to explain how they work. The ontological independence of any entity relative to its exogenous

\footnotetext{
${ }^{4}$ We owe to David Bohm one of the most accurate descriptions of the atomistic commitment, when he characterized the classical mechanistic view within Physics: "At bottom, the only changes that are regarded as possible within this scheme are quantitative changes in the parameters or functions (...), while fundamental qualitative changes in the modes of being of the basic entities and in the forms in which the basic laws are to be expressed are not regarded as possible. Thus, the essence of the mechanistic position lies in its assumption of fixed basic qualities, which means that the laws themselves will finally reduce to purely quantitative relationships" (Bohm 1984: 47, 131).
} 
relations can thus be assumed, insofar as all "extrinsic/relational/historical properties" (Kim 1999: 34) can be 'excluded', not merely from the complete description, but also from the complete characterization and explanation of any system's structure, either taken itself as a whole, or as a part of another whole.

In this context, Kim (1999: 10-12) gives the example of the alleged reduction of genes to DNA, meaning that the property 'to be a gene', functionally defined as the property of 'encoding and transmitting genetic information' or 'transmitting phenotypic characteristics from parents to off-springs', can be said to be realized by the DNA molecules. That is precisely the example that I will address (in section 5.1.), in order to exemplify my own account of a structure-integrated notion of emergence.

In sum, if interlevel emergence is definable as a double relation of partial dependence and partial independence between a fundamentally new system's macroattribute and the set of micro-attributes of its associated lower-level entities, it is mandatory to specify what attributes of the parts we should take as our reference class to consider the possible satisfaction of such kind of emergence relation. As Hempel lucidly observed, "the occurrence of a feature may be emergent with respect to one class of attributes, and not emergent relative to another class" (Hempel 1965: 260).

Given what has been said, I believe we have already one justifiable response to such question: a system is emergent from the set of its associated lower-level entities, if and only if at least one of its attributes is not produced or determined by some relation between the attributes that the lower-level entities instantiate independently of the relations in which they are involved as interacting parts or correlated relata of that very system. In this sense, my perspective is in line with Silberstein and McGeever's view that system's features are ontologically emergent when they "possess causal capacities not reducible to any of the intrinsic causal capacities of the parts nor to any of the (reducible) relations between the parts" (1999: 186), as well as with Wimsatt's claim that any notion of interlevel emergence must "involve some kind of organizational interdependence of diverse parts" (2006: 673).

In the light of what has been said so far, we can also distinguish between the relations which an entity may instantiate in a particular system but can be directly deduced from its intrinsic property types, and the relations which cannot be so deduced. In the latter case, the relations are directly connected to the new relational property types which that entity acquired by virtue of a specific structure of interactions with other entities. The same goes with micro-predicting all the attribute types of a given 
system from the attribute types that its lower-level entities instantiate independently of being interacting parts of that system.

The reason why one should focus in the relational attributes which lower-level entities may instantiate as integrated relata of particular systems should be clear. How micro-reducible or micro-determined can a system's macro-attribute be when some of the parts' micro-attributes contributing to its production are themselves instantiated by virtue of the parts' integration within that very system? How micro-explanatory can a system's macro-attribute be when some of the parts' micro-attributes to which such an explanation needs to appeal to are only, in turn, explicable by the parts' integration within that very system?

In such cases, there is no literal unilateral or asymmetric reduction of a system to its parts, nor vice-versa, but rather a reciprocal and partial determination between the lower- and the higher-levels.

\section{Dynamic relational ontology}

If microreductionism holds itself only insofar as atomistic metaphysics is also endorsed, the only way to make sense of ontological emergence by naturalistic means is to refuse the individualistic essentialism of such metaphysics. That is where a relational ontology comes into play.

Against both the atomistic view of essentially immutable individuals and the notion of wholes as primitive or self-imposed entities, I thus endorse a dynamic relational view, according to which the qualitative and causal identity of any entity, as well as its very existence and persistence conditions, must be ultimately explained as constructed and transformable by the interplay of its endogenous and exogenous relations, including those instantiated in the context of higher-level relational structures (Santos 2015a).

This view vindicates the idea that every entity is internally or necessarily causally related to other entities, both directly and indirectly. Still, this does not mean that every causal relation is internal in the sense of equally 'making a difference to', 'qualifying' or 'changing' its relata, such that they 'could not be what they are' in the absence of 
such a relation (Blanshard 1962: 472-473, 475, 486) . As Blanshard wrote, "[e]very character counts, but not all characters count equally" (1962: 482). We must, of course, acknowledge scales of intensity and degrees of relevance (1962: 485). Furthermore, as Schaffer has convincingly argued, the notion that all things are internally related in a way that renders them interdependent is "compatible with the existence of external relations, so long as there are also internal relations pervasive enough to connect all things" (2010: 372). In the most extreme scenario, only one internal relation would be enough, so long as it could be pervasive enough to connect all things. In a more temperate and reasonable sense, we may simply consider that "a plurality of partially pervasive internal relations" may connect everything together "through internal relation chains" (2010: 361-362).

Hence, to say that all things get their identity and their very existence by virtue of their relations to other things - thereby denying the main tenet of metaphysical atomism - does not mean that any set of relations will bring about a process of emergence. Many causal relations only produce quantitative changes in the already possessed attribute types of their relata, and if those changes do not exceed a certain critical limit, the relata will not undergo any qualitative kind of change (threshold effect). More than that, it is obvious that any system can instantiate some attributes which are produced by some composition of attributes which its parts instantiate independently of the relations that structure that very system.

This means that the internal or the external character of relations, the intrinsic or the relational nature of monadic attributes, as well as the contrast itself between interlevel emergence and micro-determinism can only make sense when evaluated relative to each particular system or part-whole relational context. In this sense, it is clear that any entity can instantiate some attributes independently of its relations within certain systems, although this does not mean that such attributes are intrinsic in the sense of being instantiable independently of any relations. From this it follows that we can easily acknowledge the existence of both relational and intrinsic-like monadic attributes, as well as internal and external-like relations depending on the particular part-whole contexts we are considering.

\footnotetext{
${ }^{5}$ For the fundamental difference between this notion of 'internal relation', originally elaborated by monist philosophers of the late 19th century, and the particular ways in which that notion was reformulated by philosophers such as Russell, Armstrong and Lewis, see Humphreys (2016: 103-116), and Dunn (1990a; 1990b).
} 
Being so, I propose that a given system's macro-attribute $(E)$ can only be taken as emergent, if and only if these three conditions are jointly satisfied:

1) $E$ is only instantiated by that system, taken as a distinctive ontic unit vis-à-vis the collection of its parts;

2 ) the system only instantiates $E$ by virtue of a specific global structuration (organization) of certain micro-attributes instantiated by its parts; and

3) at least one part instantiates one of such micro-attributes because that part was either transformed or generated as such from previous processes of transformation by the macro-structured network of relations constituting that system.

The latter condition allows, of course, for different degrees of dependence of the parts on their system's relational structures. The parts may depend in terms of their identity or their very existence. Likewise, a system may manifest different degrees of integration, since the dependence relation can involve many of its parts and, at the extreme, all its parts. Emergence thus comes in degrees. One way or another, interlevel emergence requires (i) the occurrence of causal relations of transformation between the parts of a given system, and (ii) that such transformations take place by virtue of a structural interdependence between those parts. In such cases, it is clear that microdeterminism and micro-reductionism fail in principle, because the lower-level entities are themselves already partially determined by the higher-level systems of interactions integrating them ${ }^{6}$.

Condition (i) confronts directly the atomistic tenet that no exogenous relation can change the qualitative or causal identity of its relata. Following (Santos 2015a), a relation is said to be transformative, if and only if it explicitly changes its relata ( $a$ ) either in terms of the instantiation of some new qualitative-structural property, with the correlative acquisition of its associated new causal power(s) of interaction, or $(b)$ in terms of the manifestation of some already possessed causal power, since the qualitative-structural property that grounds such capacity was already instantiated.

\footnotetext{
${ }^{6}$ In this sense, my relational-transformative account of emergence differs from the transformational account that has been proposed by Humphreys (2016), Guay and Sartenaer (2016) and Sartenaer (2018), given their essentially 'flat' ('same-level' or 'inter-domain') perspective. Furthermore, such flat accounts do not consider the occurrence of relations or interactions as a necessary generating source of transformational processes of emergence. More recently, Anjum and Mumford (2017) also advocated a causal-transformative model of emergence, which is generally in line with the view proposed in Santos 2015a, albeit presented in the framework of their specific version of powers ontology and theory of causation.
} 
For someone that considers only the possibility of a change of the system's parts in terms of the powers that they may manifest in some systems (e.g., Shoemaker 2002; O'Connor \& Wong 2005), some kind of individualistic essentialism is still preserved, but micro-determinism and micro-reductionism are not. In such a view, one can argue that all attributes which an entity may instantiate as a proper part of some system are nothing but intrinsic potentialities which that entity already-and-always carry with it, since it first came into existence. Relations only trigger their manifestation.

This corresponds to a preformationist form of atomistic or individualistic essentialism (PA). Still, by itself, PA does not obliterate, but on the contrary highlights, the causal efficacy of relations in the construction of the actual identity of each entity. In other words, PA cannot deny that entities do change their own actual identities because of their exogenous relations and interactions. In this sense, PA recognizes the difference-making status of relations both for the actual architecture of our world and for its scientific explanation. For without such relations the lower-level potentialities would remain latent and inactive for all eternity, never coming into actual existence, and never thus introducing the causal differences that they actually introduce in the structure and dynamics of our world.

Second, even if we admit (although we cannot really prove) the extremist metaphysical hypothesis that all potentialities are given at the outset, we must account for the why-question. It is true that if an entity instantiates a new attribute it is because it can (we owe this intuition to Aristotle). The question is rather why some potentialities are activated in some relational contexts of interactions and not in others, or why some of them are not even ever actualized. By itself, PA has no answers to such questions. And this ultimately means that, one way or another, we have to acknowledge that relations are additional ontic factors not contained in the putative list of the original potentialities in the construction of the actual identity of every single entity.

But why must we endorse the extremist hypothesis that any entity carries with it all of its potentialities since it first came into existence? On the contrary, I uphold that the relational construction of each individual entity may affect both its actual identity - that is, the attributes that it actually instantiates and exercises -, as well as its potential identity, that is, the set of attributes that it can manifest at every moment of its existence. Hence, neither the actual biography of each entity is predetermined, for the very simple reason that it may acquire new attributes, in the course of its development, by virtue of its relations with other entities, nor is any particular entity limited, ab initio, to a pre- 
given and fixed number of potentialities, since the instantiation of each new property may bring with it further causal capacities.

In this respect, I side with Gillett (2016) when he considers that entities may acquire new ('differential') causal powers as interrelated parts of certain systems, and not merely manifest their micro-latent powers as essentially intrinsic properties. Nevertheless, I diverge from Gillett when he argues that the acquisition of the new powers is to be explained in terms of an essential opposition between complex and simpler collectives, and their respective laws. In my view, the crucial distinction lies on whether the relations which structure a particular system transform their relata, regardless of that system being simpler or more complex than any other?

In this sense, the proposed view can be formulated along the lines of Borghini and Williams' (2008) dispositional modal actualism, based on a fundamental distinction between first-degree and higher-degree $(n+1)$ dispositions, the latter referring to "dispositions for the having of further dispositions" (2008: 30, n. 21). In this view, second-degree dispositions are dispositions that an entity is able to manifest if and only if a first-degree disposition manifests itself, and $n+1$ degree dispositions are dispositions that an entity may acquire if and only if an $n$ degree disposition manifests itself. If every entity has some dispositions or causal powers, and if the manifestation of any disposition brings about a change, and thus the instantiation of a new property, each manifestation of a disposition "brings into being the existence of other dispositions" (Borghini 2016: 170). This dispositional theory of possibility thus supports the existence of a "series of branching possibilities, each for some manifestation" (2008: 32) and, in that sense, it can account for cases in which the properties and the dispositions of individuals change through time (Borghini 2016: 168).

Condition (ii) allows us to grasp the idea that if each system's part is causally dependent on the other parts, in terms of their respective activities and causal contributions to the overall state and evolution of their system, each individual part is continuously conditioned by the overall structured network of relations in which it is integrated. The macro-structured network of relations cannot thus be decomposed into,

\footnotetext{
${ }^{7}$ Against the 'Simple view of aggregation', Gillett endorses "the scientific emergentist's 'Conditioned view of aggregation', which implies components have some powers in complex collectives that they would not have if the laws or principles applying in simpler collectives exhaustively applied in the complex aggregation" (2016: 17-18).
} 
or reduced to, a set of ontologically independent micro-relations. Moreover, this structural conditioning not only constrains (by restricting), but also enables (by empowering) each of the parts, in terms of the causal powers and actual activities which it may instantiate in that very system ${ }^{8}$.

This notion of structural conditioning converges with the ontic sense of the notion of contextuality as elaborated by Silberstein (2017). In such a view, contextuality is conceived as "a particular confluence of circumstances that produce a combination of constraints and stability conditions", which will in turn "limit the modal space available to the system (reduce its degrees of freedom) and open up new possibility spaces closed off outside of that context (add new degrees of freedom)" (2017: 167).

The satisfaction of the above-mentioned two conditions for interlevel emergence (viz., causal transformation and structural interdependence) thus represents the failure of the idea that every mereological complex is micro-determined and micro-reducible in terms of some composition between the intrinsic properties, and respective relations, of its compositionally or historically associated lower-level entities. From an epistemological point of view, this notion of interlevel emergence only signals the need for an interlevel integrative model of explanation.

As with the Silberstein's notion of contextual emergence, the "arrow of determination and explanation" is not exclusively bottom-up but 'multi-directional', since any causal process is bounded by relational constraints which can be top-down, bottom up, side to side (as it were)" (2017: 167-168). In this sense, I side with Silberstein when it comes to denying the existence of discrete causally closed and absolutely autonomous levels or scales of reality. In a fundamentally dynamic relational view, there is rather a relation of mutual integration, interdependence and reciprocal conditioning between the different levels (Silberstein 2017: 167), and that is the reason why "the best explanations often involve multiscale contexts" (Ibid., 187-188).

Now, are there systems which actually exemplify the two above-mentioned conditions for interlevel emergence? Yes, I think there are. They are the systems which I will call, following Bechtel \& Richardson (2010), integrated systems. That will be the subject of the next section.

\footnotetext{
${ }^{8}$ I borrow this notion of 'structural conditioning' from Margaret Archer (1995).
} 


\section{Emergence in Integrated Systems}

One of the first distinctions that we immediately meet when considering the nature of a mereological complex is that between merely scattered assorted sums of disparate entities (indistinctively called aggregates, sets, collections, or arbitrary mereological sums) and organized wholes. Unlike aggregates, the identity, existence and persistence conditions of organized wholes are only fully determined by taking into account the global structural organization of its parts (Simons 1987: 214-215, 324-32). Consequently, any non-aggregative system will possess a set of attributes - qualitative properties, causal powers and actual activities - by virtue of its specific organisational structure. From this perspective, any organized system must be analysed in terms of two fundamentally distinct dimensions:

(i) The system's micro-compositional structure, which corresponds to the set of the system's proper parts, in terms of their own individual properties and respective local relations; and

(ii) The system's macro-relational structure, which corresponds to the global organization of the different micro-properties and micro-relations of the system's parts.

As Peter Simons says,

"[a structure is] the total or overall relation of all the parts, as distinct from the multitude of binary and other relations between and among the parts. In fact, we need even to consider the various kinds and properties of the individual parts as part of this overall relation or pattern. In algebra or model theory, it is usual to define a 'relational structure' as a sequence $<D, R_{1}, R_{2}, \ldots>$ where $D$ is the domain (set of parts) and $R_{1}, R_{2}$, etc. the specific relations among these elements. The structure is here as it were a sort of super-relation on the domain and relations" (Simons 1987: 355-356).

This notion of macro-relational structure (MRS) matches the notion of 'organization' employed by the new mechanistic approach. In contrast with an aggregative compound, a mechanism is generally conceivable as a dynamic relational system whose global organization of different component parts and respective activities or interactions is taken to be responsible for a given systemic behavior, as the 
phenomenon to be explained ${ }^{9}$. Mechanisms thus have "both a 'vertical' (constitutive) and 'horizontal' (causal) dimension": mechanisms as wholes do what they do because of the global organization or orchestration of the activities of their parts (Glennan \& Illari 2018: 2).

Still, causal actions/interactions are not the only relations involved. A mechanism's MRS is a specific organization of different types of local relations between its working parts, including "spatial (e.g., location, size, shape, and motion), temporal (e.g., order, rate, and duration), and active or otherwise causal (e.g., feedback or other motifs of organization) relations to one another such that they work together" (Povich \& Craver 2018: 186). As Glennan emphasized, while the primary form of organization in a mechanism is causal, there are other forms of organization which "promote, inhibit, and guide causal interactions", such as the "spatial and temporal organization in physical, chemical, and biological mechanisms to social and cultural organization in the mechanisms described by the social sciences" (Glennan 2017: 23 and 238).

This distinction is however insufficient. In a natural continuum of intermediate cases, a further distinction was applied to two different kinds of organized systems, in terms of the nature of their constitutive relations and structures: component systems and integrated systems (Bechtel \& Richardson 2010: 2010: 26). Component systems are characterized by structures of essentially quantitative-combinatorial relations, that is, lower-level relations that keep essentially intact the qualitative and causal identity of their relata. This explains the degree of quasi-independence of the parts relative to their intra-systemic relations. As Herbert Simon said, the "intra-component linkages are generally stronger than intercomponent linkages" (Simon 1962: 477). In other words, the "behavior of the parts is intrinsically determined" (Bechtel \& Richardson 2010: 26). Simon called them 'near-decomposable systems' or 'nearly decomposable structures' (Simon 1962), while Richard Levins called them simply 'composed systems' (Levins 2017). In such systems, like an engineer's circuit, "it is feasible to determine component

\footnotetext{
${ }^{9}$ The notion of mechanism can be defined as "a structure performing a function in virtue of its component parts, component operations, and their organization. The orchestrated functioning of the mechanism, manifested in patterns of change over time in properties of its parts and operations, is responsible for one or more phenomena" (Bechtel \& Abrahamsen 2010: 323). Alternatively, Glennan and Illari proposed the following minimal notion of mechanism: "A mechanism for a phenomenon consists of entities (or parts) whose activities and interactions are organized so as to be responsible for the phenomenon" (2018:2).
} 
properties in isolation from other components, despite the fact they interact", and to determine separately the causal contributions of each part to the overall behavior of the system (Simon 1962: 474; Levins 2017: 76; Bechtel \& Richardson 2010: 199-202) ${ }^{10}$. Although the organization of the system is critical for the functioning of the system as a whole, it only provides "secondary constrains on the functioning of constituents" (Bechtel \& Richardson 2010: 26).

On the contrary, integrated systems are characterized by structures of essentially transformative and interdependent relations. In such systems, as Bechtel and Richardson emphasized, the parts are no longer intrinsically determined, but are rather conditioned and determined by their relations of system-wide interdependence, which typically involve a form of cyclic causation characteristically realized by networks of multiple feedback causal loops (2010: 25-27, 202-203). That is the reason why the structural interdependence can be said to determine, functionally, the 'significance' of the parts' activities and interactions for the system behavior (2010: 25-27) ${ }^{11}$.

\footnotetext{
${ }^{10}$ According to Levins, an engineer's circuit is a system in which the way the different parts (viz., units, condensers, transistors, wires, switches, etc.) are interrelated determines the system's properties. Therefore, the properties of the system will not be derivable from simple statistics of the properties of individual parts, as it typically happens in the case of aggregate systems. In this kind of systems, the individual parts, although physically interacting with each other, "affect the properties of the whole only by virtue of being part of a mean, or a variance, as a part of frequency" (Levins 2017: 75). On the contrary, in simply composed or component systems, structure or organization matters. Still, "the properties of the parts do not affect the mode of response of each other, but only the way in which a signal is processed that passes through all of them" (Levins 2017: 76).

${ }^{11}$ Given the notion of integrated system, it is clear that the more the parts' activities are mutually interconnected and interdependent, the more intertwined or entangled they become, and the less "successful is a sequential account of the mechanism in which each operation is treated as independent of the others" (Bechtel \& Richardson 2010: xxxv). Therefore, it is natural to find some systems which seem to defy the classical mechanistic strategies of decomposition and localization. In such cases, the explanation of the system can only be referred to the global organization of its parts (2010: 27, 32), since parts "do not seem individually to contribute to anything of interest to understanding the behavior of the whole" (2010: 202-203). This fact raises, certainly, the problem of the limits of any mechanistic account, since the behaviour of those systems only seems to be accountable for by means of dynamical mathematical explanations, which do not refer to the micro-level interactions among the system's parts, but rather characterize the development of the system in terms of its own higher-level variables. Silberstein \& Chemero (2013) argued that, in systems neuroscience, explanations are not grounded on decomposition and localization, but rather on mathematical explanations in a more lawlike fashion. The question is then whether such alternative forms of explanation conflict with each other or can rather be seen as complementary. Bechtel, for example, has been defending the need of a new dynamic mechanistic explanation. I cannot, of course, pretend to have a conclusive answer to such a question. Still, I tend to favour the second alternative since the critical issue seems to me dependent on possible scientific and technological future developments (Bechtel \& Richardson 2010: xxxv-xxxvi; Kaplan 2018).
} 
Although emphasising that such distinction is not a sharp one, since there are various degrees of interdependence, and despite his intransigent defense of an ultimately possible additive analysis of any kind of whole, Ernest Nagel did not hesitate to acknowledge that

"there is no doubt that in many systems [physical, chemical, biological, and psychological] the constituent parts and processes are 'internally' related, in the sense that these constituents stand to each other in relations of mutual causal interdependence. (...) What is distinctive of such systems, therefore, is that their parts do not act, and do not possess characteristics, independently of one another" (Nagel 1961: 392-393, 391).

For this reason, such systems were variously called 'organic' or 'functional' wholes (Nagel 1961: 380-397), 'dependence systems' (Rescher \& Oppenheim, 1955: 95), ‘evolved systems’ (Levins 2017: 76), ‘evolving systems' (Wimsatt 2007: 186-189, 213-218), or 'integral', 'cohesive' and 'integrated' systems (Simons 1987: 324-360; Mahner \& Bunge, 1997: 26; Bechtel \& Richardson 2010).

In the light of what has been argued so far, we can thus distinguish between two different kinds of macro-relational structures (MRS) that may constitute any organized system:

(i) An atomistic MRS is a global organizational relation of a number of merely quantitative-combinatorial relations between attributes, which each part instantiates (in situ) independently of being an interacting part of that structure. Parts are only determined in terms of the quantities of their intrinsic or independent properties. This means that any attribute of the structure is an attribute of a macro-structured network of fundamentally quantitative-combinatorial and independent micro-relations.

(ii) An integrated MRS is a global organizational relation of some transformative and interdependent relations, and therefore of some attributes which each part only actually (in situ) instantiates by virtue of interacting in that very structure of relations. This means that at least some of the structure's attributes are only produced or

The limits of the mechanistic approach must be thus taken as an open empirical question. We cannot settle the question a priori (still, see: Silberstein, forthcoming). 
determined by a macro-structured network of transformative and interdependent microrelations.

Now, as Richard Levins stressed, the evolved or integrated systems, the composed systems, and the aggregate systems "are obviously sufficiently different so that we must proceed with great caution in attempting to transfer ideas from one to another" (2017: 76). Still, as Wimsatt notes, a "major confusion in discussions of reductionism" arises from a "conflation" of such kinds of systems (Wimsatt 2006: 669).

In the light of this distinction, Bechtel and Richardson (2010) proposed a general notion of emergence which converges with the one here proposed. There are two basic conditions for a mechanistic notion of emergence which is "neither weak nor epistemic" (2010: xlv). First, the activities or operations of the system's parts depend on the actual behavior and the causal capacities of other parts in a cyclic (non-sequential) and nonlinear way, and "to the extent that feedback is systemwide, these dependencies will result in operations that are specific to the system". This condition refers to the fact that "the behavior of the components is system dependent" (2010: xlvi). On the other hand, this system-wide interdependence of the parts determines the system's behavior as a whole. In other words, the system is specifically defined by attributes of certain structures of 'internal' (transformative and interdependent) relations between its components. As Bechtel and Richardson argued, "[w]hen these two conditions are met, the systemic behavior is reasonably counted as emergent, even though it is fully explicable mechanistically" (Ibid.).

In an integrated structure the lower-level parts may thus instantiate new qualitative properties and/or causal powers by virtue of their interactions with the other system's parts. Still, their different causal relations do not occur or develop independently of each other. In fact, if the parts of integrated systems are said to be interdependent, so are their actions, interactions or relations, since in no meaningful way we could separate an interacting entity from its actions or relations. This is why integrated systems are considered to be only 'minimally decomposable', as it is impossible to ascribe 'independent' functions to their parts (Bechtel \& Richardson 2010: 27, 31). In other words, relations do not come one-by-one, acting separately from the others, and affecting one-after-another each interactant at a time. This, in fact, corresponds to the view which Donati and Archer refer to as the new "flat ontology" (or "single-level ontology"), widely disseminated in the contemporary social sciences, in which dyadic relations play the role of new kinds of atoms, and from whose 
combinations one should be able to reconstruct and explain all social reality in its structure and dynamics. In this new atomism of dyadic relations, "in which networks remain linkages between nodes instead of networks of relations", we are thus unable "to explain the context in which relationships occur despite there being no such thing as context-less action" (Donati \& Archer 2015: i, 22-23).

As working parts or correlated relata of an already formed integrated system, the lower-level entities are already transformed entities, being continuously, i.e., at each synchronically determined moment of their development, conditioned by the global structured network of the different local relations, each of them involving, directly or indirectly, each one of the parts. The reason why such conditioning cannot be accounted for by the mere addition of independent pairwise relations is that relations are themselves related in a web of reciprocal influences. This is the reason why parts stand (to recall Nagel's words) in 'relations of mutual causal interdependence', being 'internally' related to each other. In short, in integrated systems, the different relations are themselves related in a systemwide, structural way.

Moreover, since any macro-structured network of relations goes well beyond (or transcends) the domain of the direct, pairwise relations of each part, that structured network is realistically conceivable as being at a higher-level than each of its parts, since it interrelates and permeates all local relations, thereby covering the totality of the relata as proper parts of one common system. If an integrated system is just an organized set of relations, to say that a whole or system conditions its parts is to say that each part, taken as a relatum, is conditioned by the organized set of relations which involves it. To be conditioned is a primarily distributive property. As a matter of fact, both a system, taken as a whole, and its relational structure, belong to the same macro or higher-level vis-à-vis its component parts. The only difference is that the term 'system' (or whole) corresponds to an objectual representation of a relational system viewed, so to say, from an external, phenomenal perspective, while the term 'relational structure' (or macro-structured network) represents the same system from a relational point of view, viewing the system, so to say, from an inside perspective.

In this sense, emergent systemic attributes must be taken as inherently structural, although not in the atomistic-combinatorial sense of the notion of structure endorsed by O'Connor \& Wong (2005) following Armstrong and Kim. As I argued, the only way to make sense of the idea that a macro-structural attribute is micro-reducible to a set of micro-properties and micro-relations is that the macro-structure can itself be 
ontologically decomposed into a collection of separate and independent microproperties and micro-relations. But that is not the case with integrated systems' relational structures. In this kind of systems, at least some of the micro-attributes which causally contribute or constitute the system's structural and causal identity only become instantiated by the parts precisely by virtue of being interacting parts of that very system's global structure of relations. That is why they are 'internally related' to each other. And that is the reason why you can explain any emergent structural attribute as the structural effect of a macro-network of local relations, but you cannot reduce it to a set of ontologically primary and independent local relations. What we have is a reciprocal relation of co-determination between each part of the system and the global structure of relations in which it is involved.

An emergent structural attribute is thus neither identifiable with a mere conjunction of the attributes of the system's parts, nor with a literally indecomposable property, as O'Connor \& Wong (2005) put it. As Humphreys rightly observed, if the components of some system are internally related to each other, "the 'components' of the individual possessing the structural property are not the same inside the relation as they are outside it, and it is in virtue of those changes that the 'structural' property obtains" (2016: 245). Therefore, only if we conceive a structural relation as an external relation, "we retain the purely structural, combinatorial aspect that is intended by [O'Connor and Wong's] definition" (2016: 245). Still, for this very reason, it seems fair to note that only this combinatorial notion of structural property "fits the tradition of generative atomism applied to properties" (Humphreys 2016: 241).

An emergent structural attribute can thus be conceived as a unified complex attribute, in the sense of being an attribute of a structured network of interdependent 'internal' relations between the parts or relata of such network. In this sense, an emergent structural attribute can be said to be realized, in a dimensioned way (Gillett 2002), by the structure-dependent relations, or relational properties, of the system's parts. Taken as a whole, that relational network simply 'exhaustively constitute' the system's emergent attribute. Indeed, those are simply "two ways of describing the same thing" and "two levels of description that apply to one and the same object" (Povich \& Craver 2018: 187) ${ }^{12}$.

\footnotetext{
12 This is why there is no substantial difference between the one-one (e.g., Wilson 2015; Baysan \& Wilson 2017) and the one-many (Gillett 2016) readings of emergence relation. In my view, an emergent systemic attribute is both emergent from the collection or plurality of the parts in
} 


\subsection{The cell as a paradigm for integrated systems}

Let us take the formation process of the structural and functional identity of a eukaryotic cell as a prototypical example of an integrated system.

Today we know that eukaryotic cell's gene expression is not the product of just one cell's special component kind, like the DNA molecules, nor the outcome of a unilinear and sequential process whereby each part acts on the results of previous operations in a linear progression (i.e., decoding of DNA's information then RNA translation then functional proteins' synthesis) as a clocklike machine (Levins and Lewontin 2007: 82-83). The formation process of the structural and functional identity of a eukaryotic cell involves, instead, a network of multiple feedback causal loops which recursively affect each part's operation within a complex nonlinear dynamic (Lewontin \& Levins (2007: 82-83). For example, although it takes DNA and RNA to produce proteins, it takes proteins (e.g., transcription factors) to regulate the activity of DNA and RNA, and even to manufacture (e.g., RNA polymerases) the very nucleotide sequences “encoding” proteins (Atlan 2011: 149-150). Protein activity is thus "at once a determinant and an effect of the state of the cell" (Atlan 2011: 160). Furthermore, this circular relationship is "an ongoing process strongly influenced by ncRNA, symbiotic microbiota and environment" (Cohen, Atlan, Efroni 2016: 10). Finally, RNAs, proteins, and metabolites interact with DNA sequences "to regulate the timing, rate, and location of the cell's conversion of DNA sequence information into protein", and "much of this feedback is a consequence of physical changes in the organism that flow from developmental events themselves" (Levins \& Lewontin 2007: 82-83). These epigenetic networks thus have a retroactive effect on the state of activity of the DNA and RNA" (Atlan 2011: 150), and "in the regulation of transcription" itself (Keller 2001: 299).

Another clear example of such cyclic causal relations is the correction of transcription errors. Indeed, it is now known that transcription errors are often successfully edited by quality control mechanisms, involving a variety of proofreading mechanisms (e.g., Gamba \& Zenkin 2018). Furthermore, such "quality control mechanisms do not just remove errors; rather, they recognise misincorporations, remove them and insert correct nucleotides". And this "complex process of

terms of their intrinsic properties and associated relations, as well as from the combinatorialstructural property which applies to that set. 
recognition+removal+insertion is realised by a variety of transcription fidelity mechanisms controlled by the cell" (Vecchi 2019). The translation process, itself involving chemical modifications of the amino acids, is the starting point of further transformations. Once formed, a polypeptide chain can undergo structural changes (by assuming a primary, a secondary, a tertiary and a quaternary structure) that will ultimately contribute to the emergence of the biological functions of a cell (Griffiths et al. 2008: 319-324). Then, even in its folded state, any protein may assume several functionally different conformations by undergoing several chemical modifications, such as "enzymatic cleavage, aggregation with other molecules, phosphorylation, glycosylation, methylation, binding with molecules such as ubiquitin and so forth" (Cohen, Atlan \& Efroni 2016: 2; Griffiths et al. 2008: 344). More than that, given the same folded structure, proteins may change and acquire different functions in the course of time and in different environmental contexts (Cohen, Atlan \& Efroni 2016: 3). Finally, if the timing and amount of production of various proteins is partially controlled by complex feedback systems involving cellular environment (Lewontin 2008: 47), and if the influence of proteins on genes is modulated not only by intracellular, but also by extracellular factors, one must add the environment to the intracellular network of causal loops (Cohen, Atlan \& Efroni 2016: 4).

This leads to the conclusion that the findings of the new developmental biology "point neither to cytoplasmic nor to nuclear determination but rather to a complex but highly coordinated system of regulatory dynamics that operate simultaneously at all levels: at the level of the transcription activation, of translation, of protein activation, and of intercellular communication - in the nucleus, in the cytoplasm, indeed in the organism as a whole" (Keller 1995: 29-30). The fundamental structural and functional patterns of the cellular machinery can thus be seen as a 'state machine', in which the variables are coupled together and their temporal evolution cannot be determined separately. In such a system, the transition from one state to another state is caused by changes of the state of the ensemble of its interconnected constituents, and the calculus of the system's development has to be realized in parallel by all or some of the variables at the same time (Atlan 2011: 159-163). In this sense, a cell can alternatively be viewed as an 'evolving network', that is, a "network whose structure changes as it goes about its operations" (Atlan 2011: 163). In this case, a network of biochemical reactions and transports that function like a distributed program in a parallel computer (Atlan \& Koppel 1999; Strohman 1997; Atlan 2011: 159). 
In this sense, we can understand that DNA's property of 'being part of a unit of inheritance', or the causal power of a genic nucleotide sequence 'to code for a particular chain of amino acids' and 'to contribute to a particular cell's biological function', are in fact system-dependent relational properties which DNA molecules and their nucleotide sequences acquire solely by virtue of interacting in a particular cellular network of transformative and conditioning relations with different kinds of molecular entities such as, for instance, RNAs and proteins (Strohman 1997; Shapiro 2009) ${ }^{13}$. Hence, the very definition of what a gene is "depends on the properties of the cell in which the DNA is embedded", since the properties of a cell "are at least partly determined by transcription of DNA, but, in turn, cellular properties also determine which sequences are to be transcribed, in which combinations, and in what order" (Keller 2010: 30). As Keller emphasises, "the necessary dependency of genes on their cellular context, not simply as nutrient but as embodying causal agency, is all too easily forgotten" (Keller 2001: 309). Therefore, the causal contributions of DNA molecules and genic sequences to the structural and functional identity of a cell or organism are ultimately a product of their interactions with the other cell's components, such as proteins and RNAs, and particularly, during protein biosynthesis, subsystems such as spliceosomes and ribosomes.

This very brief and selective comments of the intricacies of eukaryotic protein biosynthesis is tailored to stress that some of the properties and causal powers usually ascribed to DNA molecules and particular genic nucleotide sequences are, in fact, relational attributes which they only instantiate by virtue of a cellular network of transformative and structural conditioning relations. Consequently, the attributes which can be ascribed to a cell taken as a system - such as its phenotypic traits or biological functions, or its ability to self-replicate, to differentiate, or to form specific patterns or organs - cannot be explained by some compositional relation between the intrinsic attributes of its constituents, as proper micro-reductionism would require. This is the reason why the formation of the structural and functional identity of an eukaryotic cell

\footnotetext{
${ }^{13}$ As Lewontin has so often emphasized, "DNA is a dead molecule, among the most nonreactive, chemically inert molecules in the living world. (...) DNA has no power to reproduce itself. Rather it is produced out of elementary materials by a complex cellular machinery of proteins. Although it is often said that DNA produces proteins, proteins (enzymes) produce DNA (...). Not only is DNA incapable of making copies of itself, aided or unaided, but it is incapable of 'making' anything else" (Lewontin \& Levins 2007: 239). See also: Lewontin 2000; and Shapiro 2009.
} 
must be viewed as a genuine cyclic or recursive process of structural-relational construction, and not as an "unidirectional sequence" of cumulative actions, such as the working of an engineer's circuit, a mechanical clock or the classical sequence 'DNA $\rightarrow$ RNA $\rightarrow$ protein $\rightarrow$ function' (Lewontin \& Levins 2007: 82-83). The more realistic model thus seems to be provided, as we saw, by the notion of an integrated system taken as an evolving network of system-wide feedback relations, involving reciprocal causal transformations, constrains, and processes of distributed control and selforganisation (Cohen, Atlan \& Efroni 2016: 4-5; Bechtel \& Richardson 2010: xlvi; Bechtel 2017) ${ }^{14}$.

\subsection{Micro-macro codetermination}

In an integrated system we find, as we saw, a reciprocal relation of codetermination between its global structure of relations and each of its embedded parts. The same goes for the ultimate causal contribution of each part's action to the overall states and behaviors of its system. This means that both the causal and functional identity of each part is determined by the structured network of relations that constitute its system. From this view, we can unproblematically say that each part of an integrated system is continuously conditioned, in terms of the qualitative properties, causal powers and interactions which it may possess and exercise, by the structured network of all of its direct and indirect relations within that system. The structural conditioning of all system's parts is thus operated through the mediation of all local relations, because they reciprocally affect each other in a system-wide and order-dependent way.

This view is compatible with the new mechanistic approach, and it can be made explicit even in terms of its own terminology, if we introduce a distinction between 'composition' and 'constitution' relationships in the following way. The notion of composition refers only to what I have called the system's 'micro-compositional structure', that is, the system's proper parts with their respective attributes. The notion of constitution adds to the micro-compositional structure the macro-relational structuration of all component parts and operations. From such a perspective, one may

\footnotetext{
${ }^{14}$ The debate about the precise relations between the notions of 'constrain' and 'control', as well as about their relations with the notion itself of 'causation', although highly relevant, goes well beyond the limits and the immediate goals of this paper. Still, see, for example: Juarrero (1999: 131-162); Moreno \& Mossio (2015: 1-38); Winning \& Bechtel (2018).
} 
say that a mechanism is "exhaustively constituted" by the structured network of its parts' activities and interactions (e.g., Povich \& Craver 2018: 193). At the same time, my position is compatible with Craver and Bechtel's view that interlevel relations are examples of "mechanistically mediated effects", that is, "hybrids of constitutive and causal relations", where the causal relations are intra-level, and the constitutive relations are interlevel (Craver and Bechtel 2007: 547). One way or another, the vertical-hierarchical relationship of mereological parthood (viz., 'to be a proper part of' a whole) can be translated, without any loss of meaning, by the horizontalinterconnectedness relationship of 'being a relatum within' a structured network of relations (Santos 2015b; see below Fig. 1$)^{15}$.

This is, in a nutshell, the structural-relational way to express and make sense of the idea, for example, that "an individual stands in an internal relation to the society in which he lives" (Humphreys 2016: 105). An integrated system is just a macrostructured network of transformative and interdependent relations. Instead of saying something like 'the whole of society' determines its constituent individuals, one should rather say that each individual is transformed and continuously conditioned by the specific structured network of different social relations in which it happens to act and interact. Again, to be conditioned is a primarily distributive property. Hence, just as we may say that each human being becomes a 'socialized individual' in a specific structured network of social relations (Archer 1995: 36), we may very well also say that each atom becomes a molecularized atom in a specific structured network of intramolecular relations, each molecule (such as DNA) becomes a cellularized molecule in a specific structured network of intracellular relations (such as in an eukaryotic cell), or each nerve cell becomes a brainized neuron in a network of intra-brain relations. This is how we could treat individual entities as lower-level parts of integrated systems. Atoms, molecules or human beings do not stop to be atoms, molecules or human beings, and that is why there is always a partial micro-determination of higher-levels - a higherlevel organization cannot be just anything, that is, regardless of the entities composing it (you cannot build living cells with crystals).

From this perspective, we can also make sense of the idea that an integrated system, formed from a structured network of interactions between its parts or precursors

\footnotetext{
${ }^{15}$ From this perspective, I diverge from Gillett (2016), since his notion of downward causation (called 'machresis') is taken to be both non-causal and non-compositional.
} 
entities of its contemporary parts, can preserve an emergent ontic status vis-à-vis the collection of its proper parts. The moment some entities start interacting in a reciprocal transformative and interdependent way is the moment they stop being independent entities and start to transform and determine each other as proper working parts or correlated relata of one common systemwide network of different relations, which may gradually form a new stable structure that is the integrated system. Now, insofar as those 'internal' relations persist, the entities internalize such relations, such that in every synchronically determined moment of their development they will appear as already transformed and structurally conditioned parts of one common structured network of relations. Any process of diachronic emergence is necessarily a process of 'double morphogenesis' (to borrow Archer's 1995 felicitous expression), in the sense that by engaging in interactions that produce some change in the macro-structure of their system, the parts are also changed.

This is how one may account for the transition from a diachronic process of emergence to a synchronic emergent status. From an interlevel viewpoint, one brings the other: given that the relations of structural conditioning manifest themselves in a temporally continuous way, their effects can be observed at any time in the development of a system and its parts. Hence, a synchronic account of emergence does not necessarily take time as a variable external to a system. At every moment of its existence, each part is internally related, through a structured network of direct and indirect relations, to the other parts, because in the absence of such relations it could not be what it is, that is, it could not instantiate the attributes that it actually instantiates in that very system. In this sense, an integrated system, in virtue of its macro-relational structure, can be taken as the real instantiator of some of its properties, and the real causal agent regarding the causal powers of interaction associated to such properties ${ }^{16}$.

\footnotetext{
${ }^{16}$ In this sense, I diverge from the transformational account proposed by Ganeri (2011), since in Ganeri's view, emergent properties are instantiated by the transformed parts in their blending state, not by the system: "the elements themselves acquire new causal powers when they are in a certain state, namely the state of jointly composing a body, powers that they did not have beforehand when they were in other combinations with other elements. This is different from the view that the body as a whole has powers which none of its parts have individually. It is instead the view that the parts themselves have new powers conditionally upon their membership of the whole" (2011: 686). As Peter Simons rightly notes, it is important "to distinguish between a collection of many individuals and the one individual they compose, if they do" (2006: 599, n.4). From this perspective, my account fits the 'new object' type of response to the collapse problem as proposed by Baysan \& Wilson (2017).
} 
We can represent the relationship between a whole, taken as an integrated system, and its proper parts, in the following way. At the lower-level we have the system's parts $(a, b, c, \ldots, n)$ with their respective causal properties or activities $\left(\mathrm{p}_{1}, \mathrm{p}_{2}, \mathrm{p}_{3}, \ldots, n\right)$. The arrows $\left(r_{1}, r_{2}, r_{3}, \ldots, n\right)$ stand for the different causal relations between the parts, forming a network of feedback loops. Each part only acts the way it does by virtue of its relations with the other parts, being thus conditioned by the global structuration $(R)$ of all other parts' interactions. That is the reason why one is obliged to recognize, at each step, the feedback loops involved, and to study how all parts' actions and interactions are globally structured or organized.

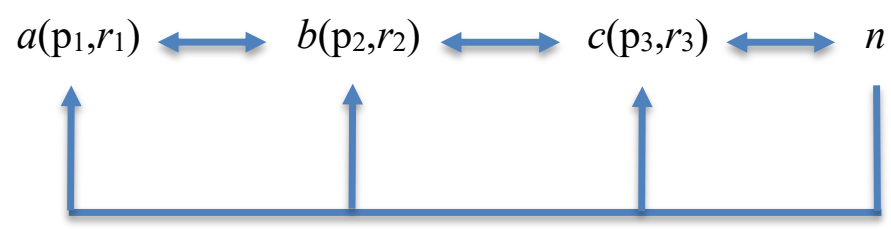

$(R)$

Fig. 1 Representation of an integrated system as a structured network of causal loops.

An integrated system (S) is just a macro-structured network $(R)$ of a set of microrelations, or relational properties, of its parts or relata: $\mathrm{S}=R\left[a\left(\mathrm{p}_{1}, r_{1}\right), b\left(\mathrm{p}_{2}, r_{2}\right), c\left(\mathrm{p}_{3}\right.\right.$, $\left.\left.r_{3}\right), \ldots, n\right]$. In the place of $\langle a, b, c, n>$ you can write DNA and RNA molecules, proteins and epigenetic factors. Instead of the classical unilinear sequence of relations 'DNA $\rightarrow$ RNA $\rightarrow$ protein $\rightarrow$ function', we get the more realistic representation of an integrated whole as an evolving network of cyclic or recursive causal loops. In fact, Fig. 1 just reproduces the general idea of the representations proposed by Strohman (1997: 197) and Atlan (2011: 150) to depict the main interactions between DNA, RNA, proteins, and epigenetic factors in the production of the cellular biological functions.

The macro-structural attributes of an integrated system are thus to be seen as causally produced and etiologically and constitutively explainable by a macrostructured network of transformative and interdependent relations between its historically or constitutively associated lower-level entities. One way or another, the mechanistic explanation of any emergent system's activity must always proceed by the 'decomposition' and 'localization' of the system's component parts and activities, by the 'recomposition' of the global orchestration of such components, and by finally 'situating' the system as a whole in its environment (Bechtel \& Richardson 2010). 
Therefore, the mechanistic notion of reduction, at least in the case of integrated systems, just refers to a necessary, but in itself insufficient, step of the explanation process. A satisfactory mechanistic explanation of an integrated system always requires an interlevel integrative account ${ }^{17}$.

\section{Conclusion}

This paper proposed an integrated-structure notion of interlevel (part-whole) emergence against the background of the micro-determinism and micro-reductionism entailed by the individualist essentialism of atomistic metaphysics.

By endorsing an alternative dynamic relational view, which assumes the occurrence of structured networks of transformative and interdependent relations, I argued that one can articulate a robust, scientific notion of interlevel emergence, which is to be found in a particular class of mereological complexes called integrated systems. Emergent structural attributes are attributes of specific macro-structured networks of transformative and interdependent relations between integrated system's parts. This is the reason why such systems cannot be explained as literal sums of their parts (as if these were essentially independent and immutable entities), but they can, and in fact must, be explained as specific modes of composition of the transformative and structurally interdependent relations of their parts.

Finally, this view thus shows the in-principle failure of micro-determinism and microreductionism, while disclosing its in-principle compatibility with an interlevel integrative neo-mechanistic model of explanation. This, however, should not come as a surprise, especially if new mechanism really represents an explicit departure from the old 'methodological' and 'metaphysical' atomism (Glennan 2017: 6).

\footnotetext{
${ }^{17}$ As Bechtel lucidly observed, "mechanistic explanations are inherently reductionistic insofar as they require specifying the parts of a mechanism and the operations the parts perform. But they also require consideration of the organization of the whole mechanism and its relation to conditions in its environment since it is only when appropriately situated that a mechanism will produce the phenomenon of interest" (Bechtel 2011: 538 - italics inserted). Therefore, "[t]he notion of reduction that arises with mechanistic explanation (...) is very different from that which has figured either in popular discussions or in recent philosophy of science, and its consequences are quite different (...). While the functioning of a mechanism depends upon its constitution, it also depends on its context, including its incorporation within systems at yet higher levels of organization" (Bechtel 2006: 40-41).
} 


\section{References}

Alexander, S. (1920). Space, Time, and Deity. London: Macmillan.

Anjum, R. \& Mumford, S. (2017). Emergence and Demergence. In M.P. Paoletti \& F. Orilia (Eds.), Philosophical and Scientific Perspectives on Downward Causation (pp. 92-109). New York: Routledge.

Archer, M. (1995). Realist Social Theory: The Morphogenetic Approach. Cambridge: Cambridge University Press.

Armstrong, D. (1978). Universals and Scientific Realism. Vol. II: A Theory of Universals. Cambridge: Cambridge University Press.

Armstrong, D. (1997). A World of States of Affairs. Cambridge: Cambridge University Press.

Atlan, H. (2011). The Genetic Program. In H. Atlan, Selected Writings. On Self-Organization, Philosophy, Bioethics, and Judaism (pp. 145-171). New York: Fordham University Press.

Atlan, H. \& Koppel, M. (1990). The Cellular Computer DNA: Program or Data?. Bulletin of Mathematical Biology, 52(3), 335-348.

Auyang, S. (1999). Foundations of complex-system theories - In economics, evolutionary biology, and statistical physics. Cambridge: Cambridge University Press.

Baysan, U. \& Wilson, J. (2017). Must Strong Emergence Collapse? Philosophica, 91, 49-104.

Bechtel, W. (2006). Discovering Cell Mechanisms: The Creation of Modern Cell Biology. Cambridge: Cambridge University Press.

Bechtel, W. (2011). Mechanism and Biological Explanation. Philosophy of Science, 78(4), 533557.

Bechtel, W. (2017). Explicating Top-Down Causation Using Networks and Dynamics. Philosophy of Science, 84(2), 253-274 (DOI: 10.1086/690718).

Bechtel, W. \& Abrahamsen, A. (2010). Dynamic mechanistic explanation: Computational modeling of circadian rhythms as an exemplar for cognitive science. Studies in History and Philosophy of Science, 41, 321-333.

Bechtel, W. \& Richardson, R. (2010). Discovering Complexity: Decomposition and Localization as Strategies in Scientific Research. Cambridge (Mass.): The MIT Press.

Bedau, M. (1997). Weak Emergence. In J. Tomberlin (Ed.), Philosophical Perspectives: Mind, Causation, and World, vol. 11 (pp. 375-399). Malden (Mass.): Blackwell.

Blanshard, B. (1962). Reason and Analysis. London: George Allen \& Unwin Ltd.

Bohm, D. (1984). Causality and Chance in Modern Physics. London: Routledge.

Borghini, A. (2016). A Critical Introduction to the Metaphysics of Modality. London-New York: Bloomsbury. 
Borghini, A. \& Williams, N. (2008). A Dispositional Theory of Possibility. Dialectica, 62, 2141.

Broad, C. D. (1925). The Mind and Its Place in Nature. New York: Harcourt, Brace \& Company/London: Routledge and Kegan Paul.

Clarke, R. (1999). Nonreductive Physicalism and the Causal Powers of the Mental. Erkenntnis, $51,295-322$.

Cohen, I., Atlan, H. \& Efroni, S. (October 2016). Genetics as Explanation: Limits to the Human Genome Project. In Encyclopedia of Life Sciences (eLS). Chichester: John Wiley \& Sons, Ltd (DOI: 10.1002/9780470015902.a0005881.pub3).

Craver, C. \& Bechtel, W. (2007). Top-down causation without top-down causes. Biology and Philosophy, 22, 547-563.

Donati, P. \& Archer, M. (2015). The Relational Subject. Cambridge: Cambridge University Press.

Dunn, J.M. (1990a). Relevant Predication 2: Intrinsic Properties and Internal Relations. Philosophical Studies, 60(3), 177-206.

Dunn, J.M. (1990b). Relevant Predication 3: Essential Properties. In J. M. Dunn and A. Gupta (Eds.), Truth or Consequences: Essays in Honor of Nuel Belnap (pp. 77-95). Dordrecht: Kluwer Academic Publishers.

Gamba, P. \& Zenkin, N. (2018). Transcription fidelity and its roles in the cell. Current Opinion in Microbiology, 42, 13-18.

Ganeri, J. (2011). Emergence, ancient and modern. Mind, 120, 671-703.

Garson, J. (2006). Emergence. In S. Sarkar \& J. Pfeifer (Eds.), The Philosophy of Science: An Encyclopedia (pp. 230-235). New York: Routledge.

Gillett, C. (2002). The Dimensions of Realization: A Critique of the Standard View. Analysis, 62, 316-323.

Gillett, C. (2016). Reduction and Emergence in Science and Philosophy. Cambridge: Cambridge University Press.

Glennan, S. (2017). The New Mechanical Philosophy. Oxford: Oxford University Press.

Glennan, S. \& Illari, P. (2018). Introduction: mechanisms and mechanical philosophies. In S. Glennan S. \& P. Illari (Eds.), The Routledge Handbook of Mechanisms and Mechanical Philosophy (pp. 1-9). New York: Routledge.

Grelling, K. \& Oppenheim, P. (1988). Logical Analysis of 'Gestalt' as 'Functional Whole' [1939]. In B. Smith (Ed.), Foundations of Gestalt Theory (pp. 210-217). Munich and Vienna: Philosophia Verlag.

Griffiths, A., Wessler, S., Lewontin R., Carroll, S. (2008). Introduction to Genetic Analysis. ${ }^{\text {th }}$ Edition. New York: W. H. Freeman and Company. 
Guay, A. \& Sartenaer, O. (2016). A New Look at Emergence. Or When After is Different. European Journal for Philosophy of Science 6(2), 297-322.

Hempel, C. (1951). General System Theory and the Unity of Science. Human Biology, 23, 313327.

Hempel, C. (1965). Aspects of Scientific Explanation and Other Essays in the Philosophy of Science. New York: The Free Press.

Hendry, R.F., Gibb, S. \& Lancaster, T. (2019). Introduction. In S. Gibb, R. Hendry, T. Lancaster (Eds.), The Routledge Handbook of Emergence (pp.1-19). London and New York: Routledge.

Humphreys, P. (2006). Emergence. In D. Borchert (Ed.), The Encyclopedia of Philosophy. Vol. 3 (pp. 190-194). New York: Macmillan.

Humphreys, P. (2016). Emergence. A Philosophical Account. Oxford: Oxford University Press. Hüttemann, A. (2004). What's Wrong with Microphysicalism?. London: Routledge.

Juarrero, A. (1999). Dynamics in Action: Intentional Behavior as a Complex System. Cambridge: MIT Press.

Kaplan, D. (2018). Mechanisms and Dynamical Systems. In S. Glennan \& P. Illari (Eds.), The Routledge Handbook of Mechanisms and Mechanical Philosophy (pp. 267-280). New York: Routledge.

Kim, J. (1993). Supervenience and Mind. Cambridge: Cambridge University Press.

Kim, J. (1998). Mind in a Physical World. Cambridge: The MIT Press.

Kim, J. (2006). Emergence: Core Ideas and Issues. Synthese, 151(3), 547-559.

Keller, E. (1995). Refiguring Life. Metaphors of Twentieth-Century Biology. New York: Columbia University Press.

Keller, E. (2001). Beyond the Gene but Beneath the Skin. In S. Oyama S, P. Griffiths \& R. Gray R. (Eds.), Cycles of Contingency: Developmental Systems and Evolution (pp. 299312). Cambridge: MIT Press.

Keller, E. (2010). It is possible to reduce biological explanations in chemistry and/or physics? In F. J. Ayala \& R. Arp (Eds.), Contemporary Debates in Philosophy of Biology (pp. 1931). Malden: Wiley-Blackwell.

Klee, R. (1984). Micro-Determinism and Concepts of Emergence. Philosophy of Science, 51, $44-63$

Levins, R. (2011). Complex Systems [1970]. In Waddington C.H. (Ed.), Organization, Stability \& Process: Towards a Theoretical Biology. Vol. 3 (pp. 73-87). New York: Routledge.

Lewis, D. (1986). Philosophical papers. Vol. 2. Oxford: Oxford University Press.

Lewis, D. (1994). Humean supervenience debugged. Mind, 103, 473-490.

Lewontin, R. (2000). The Triple Helix: Gene, Organism, and Environment. Cambridge (Mass.) and London: Harvard University Press. 
Lewontin, R. (2008). Heredity and Heritability. In S. Sarkar \& A. Plutynski (Eds.), A Companion to the Philosophy of Biology (pp. 40-57). Malden: Blackwell.

Lewontin, R. \& Levins, R. (2007). Biology Under the Influence: Dialectical Essays on Ecology, Agriculture and Health. New York: Monthly Review Press.

Mahner, M. \& Bunge, M. (1997). Foundations of Biophilosophy. Berlin-Heidelberg: SpringerVerlag.

Mill, J.S. (1974). A System of Logic Ratiocinative and Inductive, Books I-III [1843]. In Collected Works of John Stuart Mill. Vol. 7. University of Toronto Press and Routledge \& Kegan Paul.

Moreno, A., \& Mossio, M. (2015). Biological Autonomy: A Philosophical and Theoretical Inquiry. Dordrecht: Springer.

Morgan, L. (1923). Emergent Evolution. London: Williams and Norgate.

Nagel, E. (1951). Mechanistic Explanation and Organismic Biology. Philosophy and Phenomenological Research, 11, 327-338.

Nagel, E. (1952). Wholes, Sums, and Organic Unities. Philosophical Studies, 3(2), 17-32.

Nagel, E. (1961). Structure of Science: Problems in the Logic and Scientific Explanation. New York: Harcourt, Brace \& World, Inc.

O'Connor, T. \& Wong, H. Y. (2005). The Metaphysics of Emergence. Noûs, 39(4), 658-678.

Oppenheim, P. \& Putnam, H. (1958). Unity of Science as a Working Hypothesis. In H. Feigl, M. Scriven and G. Maxwell (Eds.), Concepts, Theories, and the Mind-Body Problem (pp. 3-36). Minneapolis: University of Minnesota Press.

Pettit, P. (1993). A Definition of Physicalism. Analysis, 53, 213-23.

Povich, M. \& Craver, C. (2018). Mechanistic levels, reduction, and emergence. In S. Glennan \& P. Illari (Eds.), The Routledge Handbook of Mechanisms and Mechanical Philosophy (pp. 185-197). New York: Routledge.

Quine, W. (2008). The Way the World Is [1986]. In D. Føllesdal \& D. Quine (Eds.), Confessions of a Confirmed Extensionalist and Other Essays (pp. 166-171). Cambridge (Mass.): Harvard University Press.

Rescher, N. \& Oppenheim, P. (1955). Logical Analysis of Gestalt Concepts. The British Journal for the Philosophy of Science, 6(22), 89-106.

Santos, G. (2015a). Ontological Emergence: How is that possible? Towards a new Relational Ontology. Foundations of Science, 20(4), 429-446.

Santos, G. (2015b). Upward and Downward Causation from a Relational-Horizontal Ontological Perspective. Axiomathes, 25(1), 23-40.

Sartenaer, O. (2018). Flat emergence. Pacific Philosophical Quarterly, 99(1), 225-250.

Schaffer, J. (2010). The Internal Relatedness of All Things. Mind, 119, 341-376. 
Silberstein. M. (2017). Strong Emergence No, Contextual Emergence Yes. Philosophica, 91, 145-192.

Silberstein, M. (2020). Constraints on Localization and Decomposition as Explanatory Strategies in the Biological Sciences 2.0.. In F. Calzavarini \& Viola, M. (Eds.), Neural Mechanisms: New Challenges in the Philosophy of Neuroscience. Springer Neuroscience (forthcoming).

Silberstein, M., Chemero, A. (2013). Constraints on Localization and Decomposition as Explanatory Strategies in the Biological Sciences. Philosophy of Science, 80(5), 958970.

Silberstein, M. \& McGeever, J. (1999). The Search for Ontological Emergence. The Philosophical Quarterly, 49(195), 182-200.

Simon, H. (1962). The Architecture of Complexity. Proceedings of the American Philosophical Society, 106(6), 467-482.

Simons, P. (1987). Parts. A Study in Ontology. New York: Oxford.

Simons, P. (2006). Real Wholes, Real Parts: Mereology Without Algebra. The Journal of Philosophy, 103, 597-613.

Shapiro, J. (2009). Revisiting the central dogma in the $21^{\text {st }}$ century. Annals of the New York Academy of Sciences, 1178, 6-28.

Shoemaker, S. (2002). Kim on emergence. Philosophical Studies, 108, 53-63.

Strohman, R. (1997). Epigenesis and Complexity: The coming Kuhnian revolution in biology. Nature Biotechnology, 15, 194-200.

Vecchi, D. (2019). DNA is not an ontologically distinctive developmental cause. Studies in History and Philosophy of Science Part C: Studies in History and Philosophy of Biological and Biomedical Sciences. doi:10.1016/j.shpsc.2019.101245.

Wilson, J. (2015). Metaphysical Emergence: Weak and Strong. In T. Bigaj \& C. Wüthrich (Eds.), Metaphysics in Contemporary Physics (pp. 345-402). Leiden-Boston: BrillRodopi.

Wimsatt, W. (2006). Aggregate, composed, and evolved systems: Reductionistic heuristics as means to more holistic theories. Biology and Philosophy, 21, 667-702.

Wimsatt, W. (2007). Re-Engineering Philosophy for Limited Beings: Piecewise Approximations to Reality. Cambridge (Mass.): Harvard University Press.

Winning, J. \& Bechtel, W. (2018). Rethinking Causality in Biological and Neural Mechanisms: Constraints and Control. Minds \& Machines, 28(2), 287-310. 